\title{
Gravity in the Randall-Sundrum Brane World
}

\author{
Jaume Garriga ${ }^{1,2}$ and Takahiro Tanaka ${ }^{1,2,3}$ \\ ${ }^{1}$ IFAE, Departament de Fisica, Universitat Autonoma de Barcelona, 08193 Bellaterra, Barcelona, Spain \\ ${ }^{2}$ Isaac Newton Institute, University of Cambridge, 20 Clarkson Road, Cambridge, CB3 OEH, United Kingdom \\ ${ }^{3}$ Department of Earth and Space Science, Graduate School of Science, Osaka University, Toyonaka 560-0043, Japan
}

(Received 17 November 1999)

\begin{abstract}
We discuss the weak gravitational field created by isolated matter sources in the Randall-Sundrum brane world. For the case of a single wall of positive tension, the field stays localized near the wall if the source is stationary. We calculate the leading Kaluza-Klein corrections to the linearized gravitational field of a nonrelativistic spherical object, which is different from the Schwarzschild solution at large distances. In the case of two branes of opposite tension, linearized Brans-Dicke (BD) gravity is recovered on either wall, with different BD parameters. On the wall with positive tension the BD parameter is larger than 3000 provided that the separation between walls is larger than 4 times the AdS radius. The gravitational field due to shadow matter is also considered.
\end{abstract}

PACS numbers: $04.50 .+\mathrm{h}, 11.10 . \mathrm{Kk}, 98.80 . \mathrm{Cq}$

It has recently been shown [1] that something very similar to four-dimensional Einstein gravity exists on a domain wall (or 3-brane) of positive tension which is embedded in a five-dimensional anti-de Sitter space (AdS). The striking feature about this model is that an effective dimensional reduction occurs without the need of compactifying the fifth dimension. The reason is that "Kaluza-Klein" (KK) excitations, which have nonvanishing momentum in the fifth direction, are suppressed near the brane. Thus, even though the KK modes are light, they almost decouple from matter fields - which are constrained to live on the wall. Gravitational interactions among matter fields are mediated predominantly by the "zero mode," which is often described as a bound state of gravity on the wall. The case of two parallel domain walls, one with positive tension and another with negative tension, has also been discussed in an attempt to solve the much debated hierarchy problem [2]. The possibility that we may be living in a brane is rather tantalizing, and many questions arise as to how gravity should look in such a world. What are the corrections to Einstein gravity? How does the "shadow" matter living in the other brane gravitate upon us? What is the final state of gravitational collapse? Exploring some of these aspects will be the subject of the present paper.

Some attention has been devoted to cosmological $[3,4]$, as well as nonperturbative vacuum solutions $[5,6]$ in this context. In Randall and Sundrum's solution the metric induced on the brane is flat. However, straightforward generalizations can be obtained in which the induced metric is any vacuum solution of the four dimensional Einstein's equations. Generalizations of this sort were given in [5] and [6], where the plane wave and the Schwarzschild solutions were considered. In these solutions, the metric on every spacetime slice parallel to the brane is the same as the metric on the brane, just rescaled by the AdS conformal factor. Thus, the gravitational field extends all the way to the AdS horizon, at infinite distance from the brane. In the Schwarzschild case, the five-dimensional solution is a black string hidden behind a "cylindrical" horizon extending to infinity. As shown by Chamblin et al. [6] tidal forces felt by freely falling observers actually become infinite as the AdS horizon is approached, which is not very satisfactory from the physical point of view. However, it was argued that since the infinite cylindrical horizon is unstable, the final state of collapse would perhaps have a horizon in the shape of a cigar (rather than a full infinite cylinder).

Although some intuition can be drawn from the previous examples, it would be interesting to find physical solutions where the gravitational field stays localized near the sources. For this purpose, an analysis of the weak gravitational field created by isolated matter sources on the brane seems to be the best starting point. Let us begin with the case of a single membrane of positive tension embedded in five-dimensional AdS space. The metric is given by

$$
d s^{2}=g_{a b} d x^{a} d x^{b}=d y^{2}+a^{2}(y) \eta_{\mu \nu} d x^{\mu} d x^{\nu} .
$$

Here, $a(y)=e^{-|y| / \ell}$, where $\ell$ is the curvature radius of AdS, and $\eta_{\mu \nu}$ is the Minkowski metric in four dimensions. The cosmological constant on the bulk is given by $\Lambda=$ $-6 \ell^{-2}$ and the wall tension is given by $\sigma=3 / 4 \pi \ell G_{5}$, where $G_{5}$ is Newton's constant in five dimensions.

Denoting the perturbed metric by $\tilde{g}_{a b}=g_{a b}+h_{a b}$, the Randall-Sundrum (RS) gauge is defined by

$$
h_{55}=h_{\mu 5}=0, \quad h_{\mu, \nu}^{\nu}=0, \quad h_{\mu}^{\mu}=0 .
$$

It is possible to show that these conditions can be chosen everywhere in the bulk [7]. In this gauge, the equations of motion take the simple form

$$
\left[a^{-2} \square^{(4)}+\partial_{y}^{2}-4 \ell^{-2}\right] h_{\mu \nu}=0 .
$$

The advantage of this gauge is that all components of the metric are decoupled. However, in general, when we choose the gauge (2) in the bulk, the brane will not be located at $y=0$. Instead, as we shall see, its location will be given by $y=-\hat{\xi}^{5}\left(x^{\mu}\right)$ (see Fig. 1 ), where $\hat{\xi}^{5}$ is the 
solution of the equation

$$
\square^{(4)} \hat{\xi}^{5}=\frac{\kappa}{6} T .
$$

Here $T=T_{\mu}^{\mu}$, and $\kappa=8 \pi G_{5}$. In our definition of $T_{\mu \nu}$ we are not including the contribution from the wall itself. To proceed, it will be convenient to go momentarily to Gaussian normal coordinates, which we denote by $\bar{x}^{a}$. By definition, the wall is located at $\bar{y}=0$, and we have $\bar{h}_{55}=\bar{h}_{\mu 5}=0$. Gaussian coordinates are also interesting for us because $\bar{h}_{\mu \nu}(\bar{y}=0)$ is the metric perturbation induced on the wall. We impose even parity under $\bar{y} \rightarrow-\bar{y}$, and we shall work on the positive side in the following discussion. Then, the junction condition on the extrinsic curvature at the wall requires that $\partial_{y}\left(g_{\mu \nu}+\bar{h}_{\mu \nu}\right)=$ $-(\kappa / 3)\left[\sigma\left(\gamma_{\mu \nu}+\bar{h}_{\mu \nu}\right)+3 T_{\mu \nu}-T \gamma_{\mu \nu}\right]$, which implies

$$
\begin{aligned}
&\left(\partial_{y}+2 \ell^{-1}\right) \bar{h}_{\mu \nu}=-\kappa\left(T_{\mu \nu}-\frac{1}{3} \gamma_{\mu \nu} T\right), \\
&(\bar{y}=0+) .
\end{aligned}
$$

Here $\gamma_{\mu \nu}=e^{-2|y| / \ell} \eta_{\mu \nu}$ is the background spatial metric. The condition (5) can now be expressed in the RS gauge. Since $h_{55}$ and $h_{5 \mu}$ vanish in both gauges, the most general transformation between them must take the form

$$
\begin{aligned}
\xi^{5} & =\hat{\xi}^{5}\left(x^{\rho}\right), \\
\xi^{\mu} & =\frac{-\ell}{2} \gamma^{\mu \nu} \hat{\xi}^{5}\left(x^{\rho}\right)_{, \nu}+\hat{\xi}^{\mu}\left(x^{\rho}\right),
\end{aligned}
$$

where $\hat{\xi}^{5}$ and $\hat{\xi}^{\mu}$ are independent of $y$, and for the moment we are not assuming the condition (4). From the gauge transformation equations

$$
h_{\mu \nu}=\bar{h}_{\mu \nu}-\ell \hat{\xi}_{, \mu \nu}^{5}-2 \ell^{-1} \gamma_{\mu \nu} \hat{\xi}^{5}+\gamma_{\rho(\mu} \hat{\xi}_{, \nu)}^{\rho},
$$

the junction condition (5) becomes

$$
\left(\partial_{y}+2 \ell^{-1}\right) h_{\mu \nu}=-\kappa \Sigma_{\mu \nu}, \quad(y=0+),
$$

where we have introduced the combination

$$
\Sigma_{\mu \nu}=\left(T_{\mu \nu}-\frac{1}{3} \gamma_{\mu \nu} T\right)+2 \kappa^{-1} \hat{\xi}_{, \mu \nu}^{5} .
$$

This combination, which in some sense includes the "bending" of the wall $\hat{\xi}^{5}$, will play the role of the source term in the RS gauge.

Our solutions must be even under parity, and so from (8) the derivative of the metric perturbation will be discontinuous at the wall. Combining (8) with (3), the equations of motion in the RS gauge become

$$
\left[a^{-2} \square^{(4)}+\partial_{y}^{2}-4 \ell^{-2}+4 \ell^{-1} \delta(y)\right] h_{\mu \nu}=-2 \kappa \Sigma_{\mu \nu} \delta(y)
$$

where the delta function terms will enforce the discontinuities. Of course, in order to solve (10), we must first determine the function $\hat{\xi}^{5}$, which enters in the definition of the source term $\Sigma$. This function is given by (4), as we shall now explain. Let us define the 5D retarded Green's function, which satisfies

$$
\left[a^{-2} \square^{(4)}+\partial_{y}^{2}-4 \ell^{-2}+4 \ell^{-1} \delta(y)\right] G_{R}\left(x, x^{\prime}\right)=\delta^{(5)}\left(x-x^{\prime}\right) .
$$

The formal solution of (10) is then given by

$$
h_{\mu \nu}(x)=-2 \kappa \int d^{4} x^{\prime} G_{R}\left(x, x^{\prime}\right) \Sigma_{\mu \nu}\left(x^{\prime}\right),
$$

where integration is taken over the $y=0$ surface. Since $h_{\mu}^{\mu}$ must vanish, we must impose $\Sigma_{\mu}^{\mu}=0$, which implies the "equation of motion" (4) for $\hat{\xi}^{5}$. With this choice of $\hat{\xi}^{5}$, it is easy to check that $h_{\mu \nu}$ given by Eq. (12) satisfies the harmonic condition $h_{\mu, \nu}^{\nu}=0$.

The behavior of $h_{\mu \nu}$ at infinity is determined by the form of $G_{R}\left(x, x^{\prime}\right)$. The Green's function can be constructed from a complete set of eigenstates in the usual way. Following [1], we have

$$
G_{R}\left(x, x^{\prime}\right)=-\int \frac{d^{4} k}{(2 \pi)^{4}} e^{i k_{\mu}\left(x^{\mu}-x^{\prime \mu}\right)}\left[\frac{a(y)^{2} a\left(y^{\prime}\right)^{2} \ell^{-1}}{\mathbf{k}^{2}-(\omega+i \epsilon)^{2}}+\int_{0}^{\infty} d m \frac{u_{m}(y) u_{m}\left(y^{\prime}\right)}{m^{2}+\mathbf{k}^{2}-(\omega+i \epsilon)^{2}}\right],
$$

where the first term corresponds to the zero mode and the rest corresponds to the continuum of $\mathrm{KK}$ modes $u_{m}(y)=\sqrt{m \ell / 2}\left\{J_{1}(m \ell) Y_{2}(m \ell / a)-Y_{1}(m \ell) J_{2}(m \ell / a)\right\}$ $/ \sqrt{J_{1}(m \ell)^{2}+Y_{1}(m \ell)^{2}}$. For the stationary case, it is more illustrative to consider the Green's function for the Laplacian operator, which is related to the previous one through

$$
G\left(\mathbf{x}, y, \mathbf{x}^{\prime}, y^{\prime}\right)=\int_{-\infty}^{\infty} d t^{\prime} G_{R}\left(x, x^{\prime}\right) .
$$

Here $\mathbf{x}$ are spatial Cartesian coordinates on the wall. When both points are taken on the wall $\left(y=y^{\prime}=0\right)$, we have

$$
G\left(\mathbf{x}, 0, \mathbf{x}^{\prime}, 0\right) \approx \frac{-1}{4 \pi \ell r}\left[1+\frac{\ell^{2}}{2 r^{2}}+\ldots\right]
$$

where $r=\left|\mathbf{x}-\mathbf{x}^{\prime}\right|$. Also, when one of the points is on the wall, the leading behavior for large separations in any direction is given by

$$
G\left(\mathbf{x}, y, \mathbf{x}^{\prime}, 0\right) \approx-\frac{a^{3}}{8 \pi \ell} \frac{2 a^{2} r^{2}+3 \ell^{2}}{\left(a^{2} r^{2}+\ell^{2}\right)^{3 / 2}} .
$$

This means that the metric perturbation decays rather steeply towards the AdS horizon at $y \rightarrow \infty$, i.e., $a \rightarrow 0$ (in fact even the relative metric perturbation $h_{\mu \nu} / a^{2}$ falls to zero as we move from the source). The behavior is illustrated in Fig. 1.

Since we are interested in the metric on the wall, it is convenient to transform back to Gaussian coordinates. From (7), we have $\bar{h}_{\mu \nu}=h_{\mu \nu}^{(m)}+h_{, \mu \nu}^{(\xi)}+\ell \hat{\xi}^{5}{ }_{, \mu \nu}+$ $2 \ell^{-1} \gamma_{\mu \nu} \hat{\xi}^{5}-\hat{\xi}_{(\mu, \nu)}$, where we decomposed $h_{\mu \nu}$ into 
the part corresponding to the matter fields and the part corresponding to the wall displacement,

$$
\begin{gathered}
h_{\mu \nu}^{(m)}=-2 \kappa \int d^{4} x^{\prime} G_{R}\left(x, x^{\prime}\right)\left(T_{\mu \nu}-\frac{1}{3} \gamma_{\mu \nu} T\right)\left(x^{\prime}\right), \\
h^{(\xi)}=-4 \int d^{4} x^{\prime} G_{R}\left(x, x^{\prime}\right) \hat{\xi}^{5}\left(x^{\prime}\right) .
\end{gathered}
$$

Setting $y=0$ and choosing $\hat{\xi}_{\mu}$ appropriately, we end up with the rather simple expression

$$
\bar{h}_{\mu \nu}=h_{\mu \nu}^{(m)}+2 \ell^{-1} \gamma_{\mu \nu} \hat{\xi}^{5},
$$

which gives the metric perturbation on the wall.

(i) Spherical symmetry. - As a simple application, let us now consider the effect of the KK modes on the metric. We shall restrict attention to the most interesting case of a static and spherically symmetric source, as this may be related to the final stage of gravitational collapse. With our assumptions, the energy momentum tensor can be written as

$$
T_{\mu \nu}=\rho(r) u_{\mu} u_{\nu} .
$$

From (18) with the aid of (4), we obtain

$$
h^{(\xi)}=\frac{4}{3} \int_{0}^{r} \frac{d r^{\prime}}{r^{\prime 2}} \int_{0}^{r^{\prime}} d r^{\prime \prime} r^{\prime \prime 2} V\left(r^{\prime \prime}\right),
$$

where $V=(\kappa / 2) \int G\left(x, x^{\prime}\right) \rho\left(x^{\prime}\right) d^{3} x^{\prime}$. Then, from (12), we have

$$
h_{00}=-\frac{8}{3} V(r), \quad h_{r r}=-\frac{8}{3 r^{3}} \int_{0}^{r} d r^{\prime} r^{\prime 2} V\left(r^{\prime}\right) .
$$

The remaining metric components can be found from the requirement that $h=0$ plus spherical symmetry. Notice that the fall-off properties of the metric components at $y \rightarrow \infty$ are the same as those for the Green's function (16). Hence the field decays quite steeply away from the wall. It can be checked that the perturbation of the square of the Riemann tensor behaves as

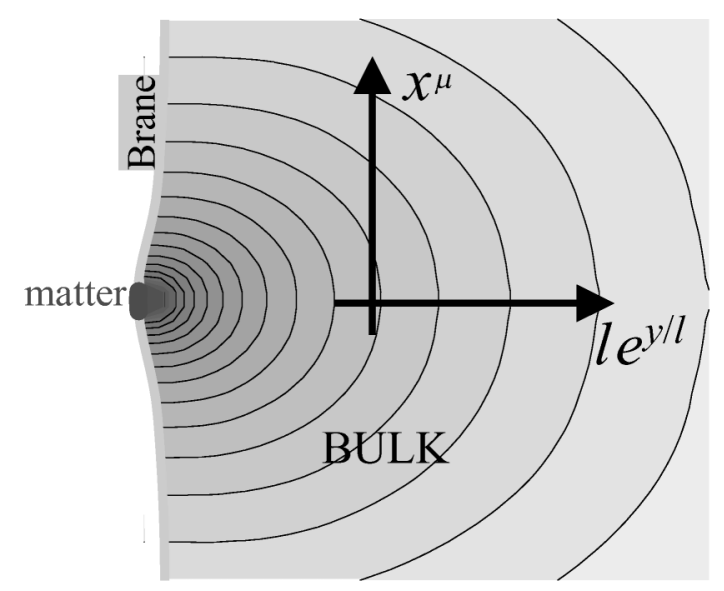

FIG. 1. Gravitational field of a spherically symmetric static source in the Randall-Sundrum gauge.

$$
\delta\left(R_{\mu \nu \rho \sigma} R^{\mu \nu \rho \sigma}\right) \propto a^{2},
$$

at large $y$ (uniformly for all values of $r$.) For comparison, the same quantity behaves as $M^{2} a^{2}(a r)^{-6}$ in the case of the Schwarzschild black string [6].

In order to find the metric on the brane, we transform to Gaussian normal coordinates. When the point is outside the source, we have

$$
V \approx-\frac{\kappa M}{8 \pi \ell r}\left(1+\frac{\ell^{2}}{2 r^{2}}\right), \quad \hat{\xi}^{5} \approx \frac{\kappa M}{24 \pi r},
$$

where $M=\int d^{3} x \rho$ is the total mass. Using (19), we arrive at the result

$$
\begin{aligned}
& \bar{h}_{00}=\frac{2 G M}{r}\left(1+\frac{2 \ell^{2}}{3 r^{2}}\right), \\
& \bar{h}_{i j}=\frac{2 G M}{r}\left(1+\frac{\ell^{2}}{3 r^{2}}\right) \delta_{i j} .
\end{aligned}
$$

It should also be stressed that the Newtonian potential $\bar{h}_{00} / 2$, which determines the attraction of neighboring bodies, is not the same as $V$-which is just proportional to the Green's function $G(x, 0)$. The coefficient in front of the correction $\ell^{2} / r^{2}$, due to the KK modes, is different in both cases, because $\hat{\xi}^{5}$ is in some sense four dimensional and contributes only to the zero mode.

Our solution differs from the weak field limit of the usual four-dimensional Schwarzschild solution. This seems to indicate that gravitational collapse of matter on the wall will not lead to a Schwarzschild black hole, but to a metric which has the asymptotic form of the weak field solution (23) [8].

(ii) Zero mode truncation. - In general, in order to obtain the metric perturbation induced on the brane, we must first solve Eq. (4) for $\hat{\xi}^{5}$, feed the solution into Eq. (10) for $h_{\mu \nu}$, and then use the gauge transformation (7) to obtain $\bar{h}_{\mu \nu}$. The expectation is that this should reproduce the results of linearized Einstein gravity with some small corrections. Let us now show that, indeed, in the case of a single brane the zero mode truncation of the fivedimensional theory coincides with the usual linearized four-dimensional gravity.

If both arguments of the two-point function are on the wall, then $G_{R}\left(x, x^{\prime}\right)$ is dominated by the zero mode contribution, $G_{R}\left(x, x^{\prime}\right) \approx \delta^{(4)}\left(x^{\mu}-x^{\mu \prime}\right) / \ell \square^{(4)}$. Substituting in (17), we find that the induced metric on the wall is given by

$$
\bar{h}_{\mu \nu}=-16 \pi G \frac{1}{\square^{(4)}}\left(T_{\mu \nu}-\frac{1}{2} \gamma_{\mu \nu} T\right),
$$

where $G=\ell^{-1} G_{5}$ is the four-dimensional Newton's constant. Thus, we recover the linearized Einstein's equations.

It should be noted, however, that a happy cancellation has occurred: the factor $1 / 3$ in Eq. (17) has turned into the familiar $1 / 2$ in the process of going to Gaussian normal coordinates (that is, absorbing $\hat{\xi}^{5}$ ) through Eq. (19). As we shall see, this cancellation does not occur in the case 
when we have two branes, which leads of course to some interesting consequences.

(iii) Two branes and light deflection. - In the case when we have two branes, one with positive tension at $y=0$ and a second one at $y=d$ with negative tension, the previous arguments can be repeated without any basic formal alterations. The only differences are that the normalization of the zero mode changes by a factor of $\left(1-e^{-2 d / \ell}\right)$ and, more important, the effect of the "Goldstone" mode $\hat{\xi}^{5}$ does not cancel out. Following the steps of the derivation given above, we find that in the zero mode approximation the gravitational field on each of the branes satisfies [9]

$$
\begin{aligned}
\left(\frac{1}{a^{2}} \square^{(4)} \bar{h}_{\mu \nu}\right)^{( \pm)}= & -\sum_{\sigma= \pm} 16 \pi G^{(\sigma)}\left(T_{\mu \nu}-\frac{1}{3} \gamma_{\mu \nu} T\right)^{(\sigma)} \\
& \pm \frac{16 \pi G^{( \pm)}}{3} \frac{\sinh (d / \ell)}{e^{ \pm d / \ell}} \gamma_{\mu \nu} T^{( \pm)}
\end{aligned}
$$

where the plus and minus refer to quantities on the wall with positive and with negative tension, respectively. Here, we have introduced

$$
G^{( \pm)}=\frac{G_{5} \ell^{-1} e^{ \pm d / \ell}}{2 \sinh (d / l)},
$$

which plays the role of Newton's constant in a BransDicke (BD) parametrization (we follow the conventions of Ref. [10]).

Strictly speaking, this parametrization holds when "the other" wall (the one in which we do not live) is empty. Let us first consider this situation. In this case, the BD parameter is given by

$$
\omega_{\mathrm{BD}}^{( \pm)}=\frac{3}{2}\left(e^{ \pm 2 d / l}-1\right) .
$$

Observations require that $\omega_{\mathrm{BD}}>3000$ [10]. In the positive tension brane, this is achieved with $d / l>4$, and we have an acceptable gravity theory even without stabilizing the dilaton. In the negative tension brane, we find that the $\mathrm{BD}$ parameter is always negative but greater than $-3 / 2$. In the Einstein frame, the kinetic term for the BD field has the usual sign for $\omega_{\mathrm{BD}}>-3 / 2$. This suggests that the system of two branes is well behaved in spite of the negative tension in one of the branes.

Now, let us consider the effect of "shadow" matter, which lives on the other membrane. This appears only in the first term in (25). Hence, for nonrelativistic matter, and assuming spherical symmetry, its contribution to the Newtonian potential $\bar{h}_{00}$ will be twice as large as its con- tribution to any of the diagonal spatial components, say $\bar{h}_{z z}$. This is in contrast with the situation in Einstein's theory, where the contribution to the Newtonian potential is the same as the contribution to $\bar{h}_{z z}$. For a source in the $x, y$ plane, the deflection of a light ray traveling in the $y$ direction is given by $\ddot{x}=(1 / 2)\left(\bar{h}_{00}+\bar{h}_{y y}\right)_{, x}$. Therefore, for the same Newtonian mass, the deflection of light rays caused by shadow matter is $25 \%$ smaller than that in Einstein gravity. It would be interesting to investigate this possible effect in an astrophysical context. This is left for future research.

This work was done while participating in the workshop on Structure Formation in the Universe, at the Isaac Newton Institute. We thank the organizers for their hospitality, and the participants for numerous conversations. We owe special thanks to M. Bucher, T. Chiba, S.W. Hawking, R. Gregory, K. Maeda, X. Montes, A. Pomarol, O. Pujolas, V. A. Rubakov, H. Reall, M. Sasaki, T. Shiromizu, and N. Turok. J. G. acknowledges support from CICYT, under Grant No. AEN98-1093. T. T. acknowledges support from Monbusho System to Send Japanese Researchers Overseas.

[1] L. Randall and R. Sundrum, Phys. Rev. Lett. 83, 4690 (1999).

[2] L. Randall and R. Sundrum, Phys. Rev. Lett. 83, 3370 (1999).

[3] P. Binétruy, C. Deffayet, U. Ellwanger, and D. Langlois, hep-th/9910219.

[4] T. Shiromizu, K. Maeda, and M. Sasaki, gr-qc/9910076.

[5] A. Chamblin and G. W. Gibbons, hep-th/9909130.

[6] A. Chamblin, S. W. Hawking, and H.S. Reall, hep-th/ 9909205.

[7] The RS gauge can be chosen in the bulk for almost the same reason that allows the choice of the synchronous tranverse and traceless gauge in the cosmological context (in the absence of matter sources). In fact, there is some residual gauge freedom generated by (6) with $\hat{\xi}^{\nu},{ }_{, \nu}, \mu+2 \hat{\xi}^{\mu}{ }_{, \nu}, \nu=0$ and $\hat{\xi}^{5}=\ell \hat{\xi}^{\mu}{ }_{, \mu} / 4$.

[8] For the $2+1$ dimensional case, some discussion of this issue is given in R. Emparan, G. T. Horowitz, and R. C. Myers, hep-th/9911043.

[9] In an earlier version of this paper the sign of the last term of Eq. (25) in the case of a negative tension brane was not correct. This was pointed out to us by V. A. Rubakov.

[10] C.M. Will, Theory and Experiment in Gravitational Physics (Cambridge University Press, Cambridge, 1993), revised ed., p. 123; in Proceedings of the 1998 Slac Summer Institute on Particle Physics (gr-qc/9811036). 